\title{
ANEURISMA DA ARTÉRIA CARÓTIDA INTERNA EXTRACRANIANA: RELATO DE CASO*
}

\author{
Marcio Ricardo Taveira Garcia ${ }^{1}$, Maria Cristina Chammas ${ }^{2}$, Ângela Hissae Motoyama Caiado ${ }^{3}$, \\ Adriana Gonçalves Juliano ${ }^{4}$, Claudia da Costa Leite ${ }^{5}$, Giovanni Guido Cerri ${ }^{6}$
}

\begin{abstract}
Resumo Os aneurismas verdadeiros da artéria carótida interna extracraniana são raros, ao contrário dos supraclinóideos, somando menos de $4 \%$ dos aneurismas periféricos. Eles se apresentam clinicamente como massas palpáveis cervicais, junto à margem inferior do ângulo da mandíbula, causando rouquidão, disfagia e dor por compressão nervosa. Há freqüente associação desta doença com outros aneurismas periféricos devido à sua etiologia principal (aterosclerose). Os aneurismas periféricos são comumente identificados à ultra-sonografia Doppler, quando na vigência de janela acústica adequada. Nesta situação, os aneurismas podem ser avaliados tanto morfológica como hemodinamicamente. Sua identificação e estudo são importantes para prevenir graves complicações, como tromboses, infartos maciços ou embólicos da área correspondente no sistema nervoso central, ruptura e dissecção, além de auxiliar na indicação da melhor conduta terapêutica. Unitermos: Aneurisma; Artéria carótida interna extracraniana; Ultra-sonografia; Ultra-sonografia Doppler; Dúplex-Doppler colorido.
\end{abstract}

\section{Abstract Aneurysm of the extracranial internal carotid artery: a case report.}

Unlike supraclinoid aneurysms, true aneurysms of the extracranial internal carotid artery are extremely rare (less than $4 \%$ of the peripheral aneurysms). The commonest presentation is a pulsatile neck swelling below the angle of the jaw associated with hoarseness, dysphagia and pain (neural compression). Concomitance with other peripherical aneurysms is frequent and caused by atherosclerosis. The morphological and hemodynamic features are very well evaluated by Doppler ultrasound, when the acoustic window is satisfactory. Identification and evaluation of these aneurysms are very important to prevent thrombosis, rupture, dissections, massive strokes and embolic brain infarcts, besides helping in the decision of the best treatment. Key words: Aneurysm; Extracranial internal carotid artery; Ultrasound; Doppler ultrasound; Color duplex Doppler ultrasound.

\section{INTRODUÇÃO}

A artéria carótida interna tem cerca de $8 \mathrm{~cm}$ de extensão em seu segmento cervical, prolonga-se desde a sua bifurcação até o forame crivoso, não se ramificando ${ }^{(1)}$. Os aneurismas verdadeiros da artéria carótida interna extracraniana são extremamente $\operatorname{raros}^{(2)}$, ao contrário dos supraclinóideos, correspondendo a menos de $4 \%$ dos aneu-

* Trabalho realizado no Instituto de Radiologia (InRad) do Hospital das Clínicas da Faculdade de Medicina da Universidade de São Paulo (HC-FMUSP), São Paulo, SP.

1. Médico Radiologista Assistente do Hospital Universitário da USP, Médico Colaborador do Setor de Tomografia do InRad/ HC-FMUSP.

2. Diretora do Serviço de Ultra-Sonografia do InRad/HC-FMUSP. 3. Médica Residente do Departamento de Radiologia da FMUSP.

4. Médica Assistente do Departamento de Radiologia da FMUSP.

5. Vice-Chefe do Departamento de Radiologia da FMUSP.

6. Professor Titular do Departamento de Radiologia, Diretor da FMUSP.

Endereço para correspondência: Dr. Marcio Ricardo Taveira Garcia. Rua Luzia Inácia Ascenso, 254, City Bussocaba. Osasco, SP, 06040-320. E-mail: Mrtgarcia@yahoo.com.br

Recebido para publicação em 23/9/2003. Aceito, após revisão, em 20/10/2003. rismas periféricos ${ }^{(3,4)}$. Esta doença deve estar entre os diagnósticos diferenciais de massas cervicais. Habitualmente apresentam-se como massas palpáveis e pulsáteis, junto às margens inferiores dos ângulos da mandíbula $^{(3,5,6)}$. Outros sintomas incluem rouquidão, disfagia, dor por compressão nervosa e alterações neurológicas decorrentes de isquemias transitórias no território cerebral ${ }^{(\mathbf{5})}$.

\section{RELATO DO CASO}

Paciente do sexo feminino, 70 anos de idade, natural de São Paulo, SP, procurou o ambulatório do Serviço de Cirurgia de Cabeça e Pescoço, da Divisão de Cirurgia, do Hospital das Clínicas da Faculdade de Medicina da Universidade de São Paulo, para avaliação de massa cervical indolor. Ao exame físico caracterizou-se massa arredondada, endurecida, apresentando pouca mobilidade, sem sinais flogísticos, não pulsátil, situada na topografia do ângulo da mandíbula, junto à extremidade inferior da glândula parótida direita. A principal hipótese diagnóstica formulada foi de nódulo parotídeo, sendo, então, solicitada punção aspirativa por agulha fina guiada por ultrasonografia no nosso serviço.

Ao exame ultra-sonográfico modo-B observou-se formação arredondada, hipoecogênica, de limites bem definidos, apresentando paredes espessadas por material ecogênico de aspecto laminar, situada no espaço carotídeo, deslocando lateralmente as estruturas do espaço parotídeo, medindo $2,7 \times 2,2 \times 2,0 \mathrm{~cm}(\mathrm{CC} \times \mathrm{LL} \times \mathrm{AP})$. Ao mapeamento colorido identificou-se vascularização exuberante no interior da lesão, com padrão de fluxo turbilhonado, contígua ao terço proximal da artéria carótida interna. Tal formação correspondia a aneurisma verdadeiro e sacular, parcialmente trombosado, da artéria carótida interna direita extracraniana. O exame do lado oposto identificou outro aneurisma de dimensões menores, de padrão fusiforme, na topografia contralateral correspondente, medindo $1,1 \times 0,8 \times 0,8 \mathrm{~cm}(\mathrm{CC} \times \mathrm{LL} \times \mathrm{AP})$. 


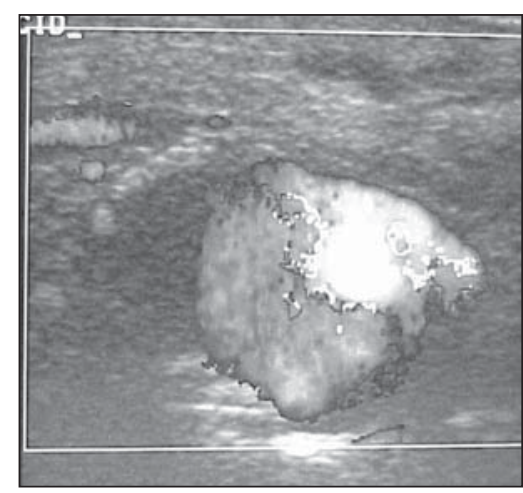

A

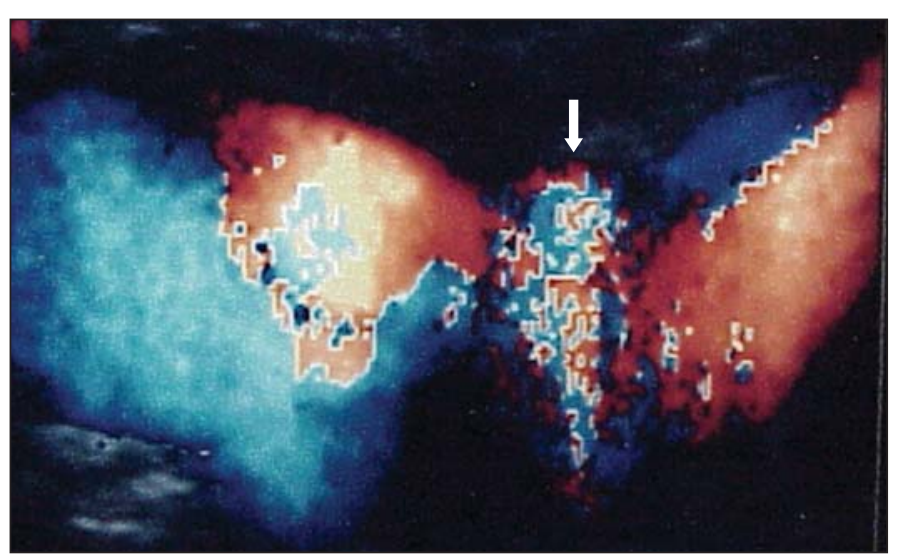

B
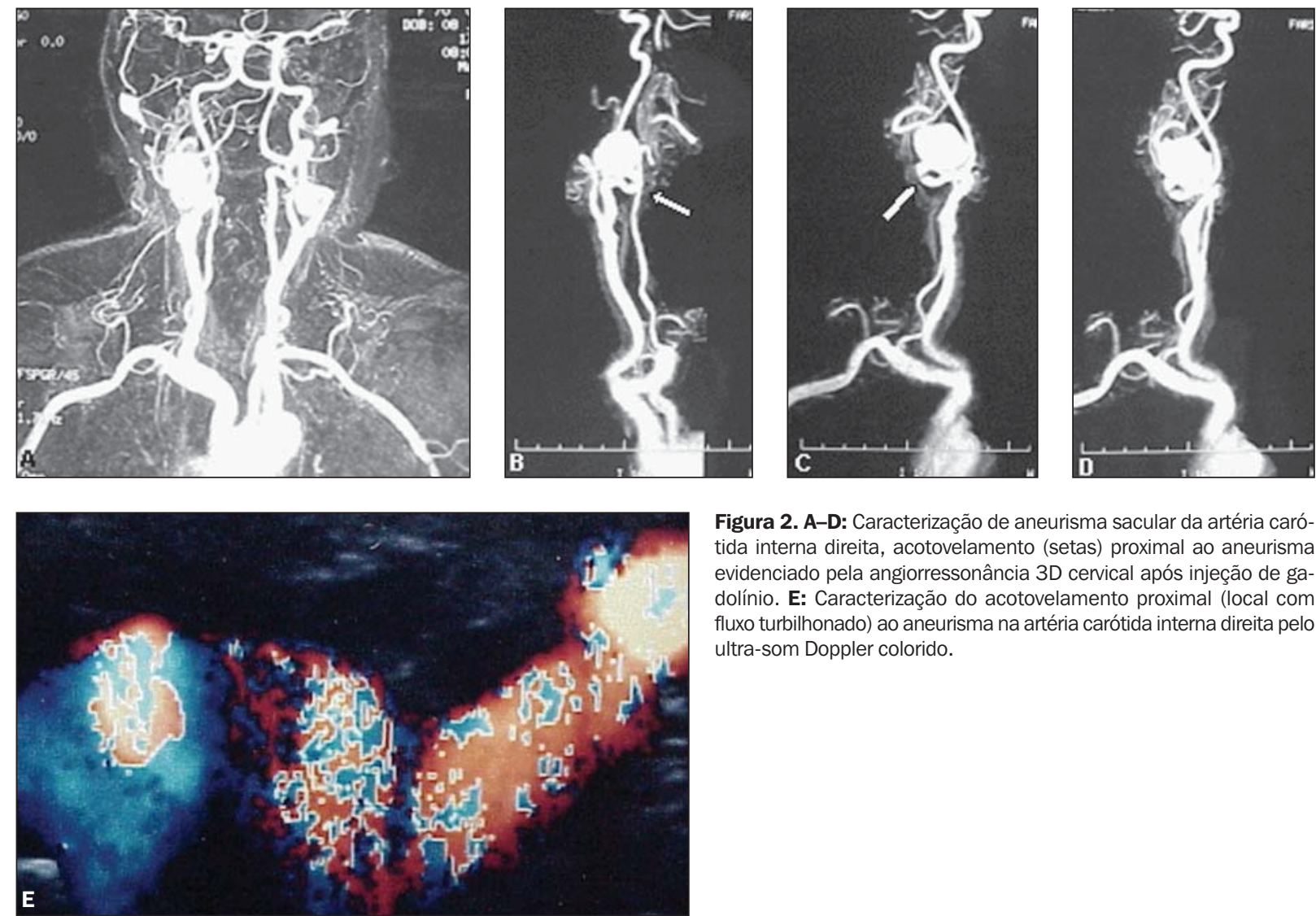

Figura 2. A-D: Caracterização de aneurisma sacular da artéria carótida interna direita, acotovelamento (setas) proximal ao aneurisma evidenciado pela angiorressonância 3D cervical após injeção de gadolínio. E: Caracterização do acotovelamento proximal (local com fluxo turbilhonado) ao aneurisma na artéria carótida interna direita pelo ultra-som Doppler colorido. 

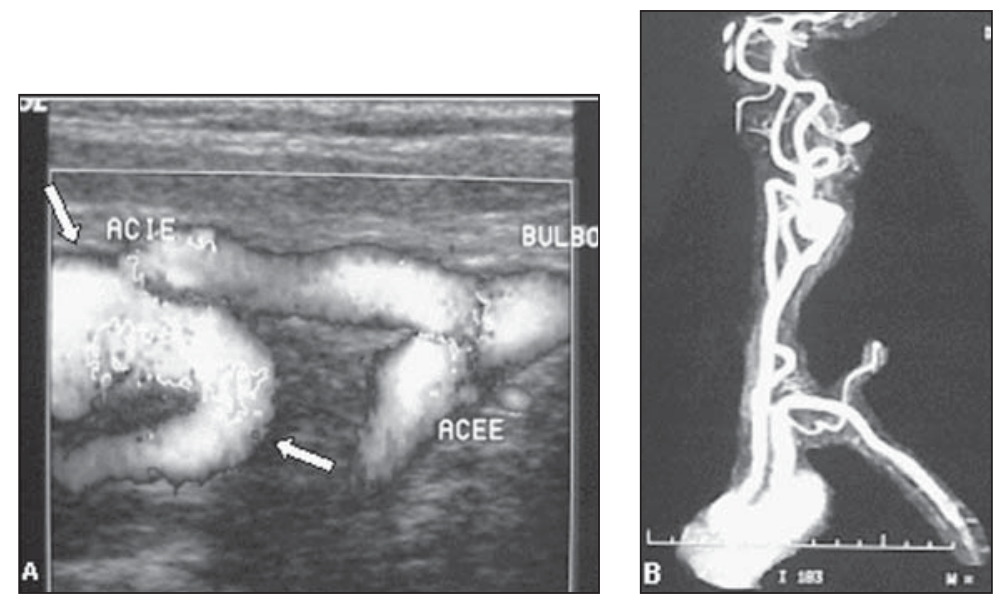

Figura 3. Aneurisma fusiforme associado a duplo acotovelamento (setas) da artéria carótida interna esquerda demonstrado pelos métodos ultra-som Doppler $(\mathbf{A})$ e angiorressonância 3D cervical após injeção de gadolínio (B).

A ressonância magnética foi realizada para pesquisa de outros aneurismas intracranianos e melhor caracterização de suas relações anatômicas. Este método confirmou os achados ultra-sonográficos e não identificou outros territórios aneurismáticos das artérias carótidas internas.

\section{DISCUSSÃO}

A artéria carótida interna tem cerca de $8 \mathrm{~cm}$ de extensão em seu segmento cervical, onde não apresenta $\operatorname{ramos}^{(\mathbf{1})}$. Os aneurismas verdadeiros da artéria carótida interna extracraniana são extremamente ra$\operatorname{ros}^{(2)}$, correspondendo a menos de $4 \%$ dos aneurismas periféricos ${ }^{(\mathbf{3 , 4})}$. Esta doença deve estar entre os diagnósticos diferenciais das massas no território cervical. Habitualmente apresentam-se como massas palpáveis e pulsáteis, junto às margens inferiores dos ângulos da mandíbula ${ }^{(\mathbf{3 , 5 , 6 )}}$. Outros sintomas incluem rouquidão, disfagia, dor por compressão nervosa e alterações neurológicas decorrentes de isquemias transitórias no território cerebral ${ }^{(\mathbf{5})}$.

Sua etiologia pode se dever a aterosclerose, displasia fibromuscular, síndrome de Marfan, trauma, cirurgia prévia no território carotídeo, infecção (aneurisma micótico), defeitos congênitos e dissecção ${ }^{(7,8)}$. A principal delas é a aterosclerose, e por isso deve-se procurar a concomitância desses aneurismas com outros periféricos, que é achado freqüente ${ }^{(\mathbf{8})}$, conforme pudemos observar no caso relatado.
Além das características já expostas na descrição do exame ultra-sonográfico, os aneurismas da artéria carótida interna extracraniana podem apresentar calcificações periféricas, o que auxilia no seu diagnóstico ao modo-B. Contudo, esta doença é facilmente confirmada quando o estudo dúplex-Doppler colorido é realizado. Outros métodos diagnósticos desta doença são a tomografia computadorizada, a angiografia (que detém o padrão ouro de avamite a construção de imagens tridimensionais, capazes de mostrar o tamanho do aneurisma, sua relação anatômica com as outras estruturas cervicais e a extensão do trombo mural, quando presente $\mathrm{e}^{(7)}$.

Sua identificação e avaliação são importantes, uma vez que suas complicações são graves. Entre as principais complicações destacam-se a trombose com infartos maciços ou embólicos da área correspondente suprida no sistema nervoso central, a ruptura e a dissecção do aneurisma, sendo indicado na maioria das vezes o tratamento cirúrgico dessas lesões ${ }^{(7)}$. A completa excisão do aneurisma não é indicada devido à alta incidência de lesão dos nervos cranianos que têm seus trajetos descendentes junto a esses $\operatorname{vasos}^{(\mathbf{7 , 9 )}}$.

Os aneurismas da artéria carótida interna são alterações incomuns na porção extracraniana desta artéria. Apesar disso, devem sempre ser incluídos nos diagnósticos diferenciais das massas cervicais. Como observamos no caso relatado, o nódulo, ao liação) e a ressonância magnética, que per- exame físico, era endurecido e não pulsátil, direcionando o diagnóstico do cirurgião para lesão de natureza parotídea. Contudo, foi de fundamental importância o pedido do cirurgião de punção aspirativa por agulha fina guiada por ultra-sonografia, que evitou o procedimento inadvertido da massa, realizou o diagnóstico e contra-indicou a punção da formação aneurismática.

A evolução do aneurisma da artéria carótida extracraniana é desfavorável devido às suas inúmeras complicações, necessitando de estudo detalhado para a indicação da melhor conduta terapêutica. Este é normalmente feito utilizando-se a ultra-sonografia, aliada ao estudo Doppler (colorido e pulsado), por sua praticidade, disponibilidade, baixo custo e características inóquas para o paciente.

O diagnóstico pode ser complementado com tomografia computadorizada ou ressonância magnética, para detalhamento da sua relação com as outras estruturas cervicais. A angiografia, no entanto, é o melhor método diagnóstico e o mais útil no planejamento cirúrgico, que pode ser aberto ou endovascular com a utilização de "stents".

\section{REFERÊNCIAS}

1. Www.sosdoutor.com.br/sosvascular/doenças carotidas.asp

2. Akan H, Selçuk MB, İncesu L, Güneş M, Tekat A. Aneurysm of extracranial internal carotid artery: radiologic evaluation. Turk A Otol 1997;35:111-4.

3. Brito CJ, Merlo I, Fonseca Filho VL. Aneurismas dos troncos supra-aórticos. In: Brito CJ. Cirurgia vascular. 1aㅡ ed. Rio de Janeiro: Revinter, 2002: 525-32.

4. Maffei FHA, Lastória S, Yoshida WB, Rollo HR. Doenças vasculares periféricas. $3^{\underline{a}}$ ed. Rio de Janeiro: Medsi, 2002:1157-60.

5. Mokri B, Piepgras DG, Sundt TM Jr, Pearson BW. Extracranial internal carotid artery aneurysms. Mayo Clin Proc 1982;57:310-21.

6. Rittenhouse EA, Radke HM, Sumner DS. Carotid artery aneurysm. Review of the literature and report of a case with rupture into the oropharynx. Arch Surg 1972;105:786-9.

7. Lau H, Cheng SWK, Lam KY. Carotid artery aneurysm secondary to cystic medial necrosis. J R Coll Surg Edinb 2001,46:173-5.

8. Rutherford RB. Arterial aneurysms: etiologic considerations. In: Rutherford RB, ed. Vascular surgery. 3rd ed. Philadelphia: WB Saunders, 1989: 238-45.

9. Liapis CD, Gugulakis A, Misiakos E, Verikokos C, Dousaitou B, Sechas M. Surgical treatment of extracranial carotid aneurysms. Int Angiol 1994; 13: 290-5. 